\title{
The Functional IgE-Blocking Factor Induced by Allergen-Specific Immunotherapy Correlates with IgG4 Antibodies and a Decrease of Symptoms in House Dust Mite-Allergic Children
}

\author{
Deyu Zhao ${ }^{a}$ Xuxin Lai ${ }^{b}$ Man Tian ${ }^{a}$ Yanhe Jiang ${ }^{a}$ Yiwu Zheng ${ }^{b}$ \\ Birgitte Gjesing $^{b}$ Nanshan Zhong ${ }^{c}$ Michael D. Spangfort ${ }^{b}$ \\ a Respiratory Department, The Affiliated Children's Hospital of Nanjing Medical University, Nanjing, and \\ ${ }^{b}$ Research Asia Pacific, ALK A/S, Guangzhou, and 'State Key Laboratory of Respiratory Diseases, First Affiliated \\ Hospital of Guangzhou Medical University, Guangzhou, PR China
}

\section{Key Words}

Allergen-specific immunotherapy · House dust mite ·

lgE-blocking factor $\cdot \lg \mathrm{G} 4$

\begin{abstract}
Background: At present, there are no validated biomarkers reflecting or predicting the clinical efficacy of allergen-specific immunotherapy (AIT). We aimed to investigate the correlations between clinical and immunological responses of patients undergoing house dust mite (HDM) AIT. Methods: Sixty-nine children diagnosed with HDM allergic rhinitis and/or asthma received standardized Dermatophagoides pteronyssinus (Dp) subcutaneous AIT for 12 months. Twenty HDM-allergic children served as an open control group. Clinical symptom and medication scores were recorded and Dp-specific $\lg \mathrm{E}$, IgG4 and IgE-blocking factor were measured before AIT and after 4 and 12 months of AIT. Results: Symptom scores decreased after 4 months and continued to decrease during 12 months of AIT. No differences in medication scores were observed between AIT and the control group during the study period. Levels of Dp IgG4 increased after 4 months and
\end{abstract}

correlated to symptom scores at 12 months $(r=-0.296, p=$ 0.013) of AIT. The Dp lgE-blocking factor increased after 4 months of AIT, and correlated with symptom scores at 4 months $(r=-0.307, p=0.010)$ and 12 months $(r=-0.288, p=$ 0.016) of AIT. A strong correlation between Dp IgE-blocking factor and Dp Ig G4 during AIT ( 4 months: $r=0.680 ; 12$ months: $r=0.636$, both $p<0.0001$ ) was observed. Patients with lgEblocking factor $\geq 0.2$ after 4 months of AIT showed lower symptom scores at 12 months of AIT $(p=0.0093)$. Conclusions: Subcutaneous HDM AIT results in a decrease of allergic symptoms among HDM-allergic children. IgE-blocking activity increased after 4 months of AIT and correlated with clinical symptoms. A high IgE-blocking factor at an early stage of AIT is associated with fewer symptoms at a later stage of AIT.

(c) 2016 S. Karger AG, Basel

\section{Introduction}

Allergen-specific immunotherapy (AIT) using standardized vaccines is an effective treatment for allergic rhinitis and allergic asthma and is the only treatment addres-

\section{KARGER}

E-Mail karger@karger.com

www.karger.com/iaa
(C) 2016 S. Karger AG, Basel

$1018-2438 / 16 / 1692-0113 \$ 39.50 / 0$ 
Table 1. Demographic data and clinical characteristics

\begin{tabular}{lll}
\hline & AIT group & Control group \\
\hline Age, years & $7(5$ to 14$)$ & $5(3$ to 9$)$ \\
Male/female & $47 / 22$ & $10 / 10$ \\
Symptom scores & $3.0(1.3$ to 5.0$)$ & $2.0(0$ to 4.0$)$ \\
Medication scores & $3.0(0$ to 8.0$)$ & $2.5(0$ to 4.3$)$ \\
Dp IgE, kU/l & $116(12$ to 667$)$ & $50(5$ to 716$)$ \\
Dp IgG4, AU/ml & $404(20$ to 6,180$)$ & $339(62$ to 1,697$)$ \\
Dp IgE BF & $-0.17(-0.81$ to 0.23$)$ & $-0.15(-0.90$ to 0.26$)$ \\
\hline
\end{tabular}

Data are presented as $\mathrm{n}$ or the median (range).

sing the underlying cause of allergic diseases $[1,2]$. AIT has the potential to modify the development of disease and has a long-term prevention effect on asthma [3]. Several studies have demonstrated that AIT induces substantial amounts of allergen-specific IgG4 $[4,5]$ with the potential to compete with allergen-specific IgE, inhibit basophil activation and prevent IgE-facilitated allergen presentation to T cells [6-10]. IgG4 antibodies do not activate complement [11] and are unable to cross-link allergens on mast cells due to its heterobivalency [12]. Apart from IgG4, other subtypes of IgG or serum components are likely to have blocking activity as well. The induction of blocking antibodies is an important mechanism underlying AIT [13]. Changes in concentrations of allergenspecific serum IgG4 following AIT is a marker for an immune response towards the administrated AIT extract. However, it is still not clear to what degree elevated levels of allergen-specific IgG4 are indicative of a successful clinical outcome for individual AIT patients. The ability of AIT-induced allergen-specific serum IgG4 and other allergen non-IgE antibodies to block the binding between specific IgE and allergens can be estimated by measuring the competition between serum components and specific IgE antibodies for allergen binding and quantified as an allergen-specific IgE-blocking factor (BF). For pollen AIT, it has recently been demonstrated that functional assays of AIT-induced serum components correlate better to a clinical and long-term persistent effect than an increase in specific IgG4 titers $[14,15]$.

House dust mite (HDM) is the dominating indoor allergen source in China and some geographical regions show a very high prevalence of HDM allergic monosensitization among patients [16]. In a previous study, we showed that, compared to adults, children treated with standardized HDM subcutaneous therapy are more responsive to AIT, demonstrating a clinical improvement and producing higher levels of allergen-specific IgG4 during a shorter AIT period [5]. AIT is recommended by international guidelines to continue for 3 years. Clinical improvements are often experienced after 3-6 months and immunological responses, such as the presence of IL-10 and allergen-specific IgG4, has been demonstrated after 3 months $[5,17]$. In this study we investigated and correlated changes in symptom and medication scores and antibody concentrations and function in children who received 1 year of HDM injection AIT.

\section{Materials and Methods}

\section{Subjects}

The study includes 69 HDM-allergic children (5-14 years of age, mean age 7 years) receiving 1 year of HDM AIT. Table 1 shows the demographic data. All patients fulfilled the ARIA guideline for allergic rhinitis and/or GINA guidelines for mild-to-moderate asthma. All patients were skin prick test positive to Dermatophagoides pteronyssinus (Dp; ALK-Abello A/S, Hørsholm, Denmark) and had serum IgE against $\mathrm{Dp}>0.35 \mathrm{kU} / \mathrm{l}$. All subjects attended the respiratory clinic in Nanjing Children's Hospital and were given the necessary palliative medication during the study year.

Due to ethical and practical considerations, the study could not be conducted as a randomized, double-blind, placebo-controlled trial. However, an open control group consisting of 20 dust miteallergic children using symptomatic medication but not receiving AIT was included and used for comparing the changes in clinical scores and immunological parameters with the AIT group. The decision to enter a subject into the AIT or control group was based on the preference of the patient and their parents.

Blood samples were collected from each patient at three time points: before treatment, at the end of the AIT up-dosing phase (4 months AIT) and after an additional 8 months of maintenance treatment $\left(12\right.$ months AIT). Sera were stored at $-18^{\circ} \mathrm{C}$.

The study was approved by the local ethical committee and informed consent was obtained from a parent or guardian of each individual participant in the study. The study is reported on the China Clinical Trial Registry (registration No. ChiCTR-OPC-15006105; http://www.chictr.org.cn/showproj.aspx?proj=10423).

\section{AIT Protocol}

The patients were treated with subcutaneous injections of standardized aluminum-formulated HDM Dp Alutard ${ }^{\circledR}$ SQ vaccine (ALK-Abello A/S). The treatment protocol followed the recommended up-dosing schedule of 16 weeks before reaching the maintenance dose of 100,000 Alutard ${ }^{\circledR}$ SQ given every 6-8 weeks for 3 years. The data in this study was collected during the first year of AIT treatment.

\section{Clinical Response}

Patients were asked to rate their symptoms of asthma (day and night; including wheeze, cough, chest tightness and shortness of breath) and rhinitis (over the whole day; including nose itching, 


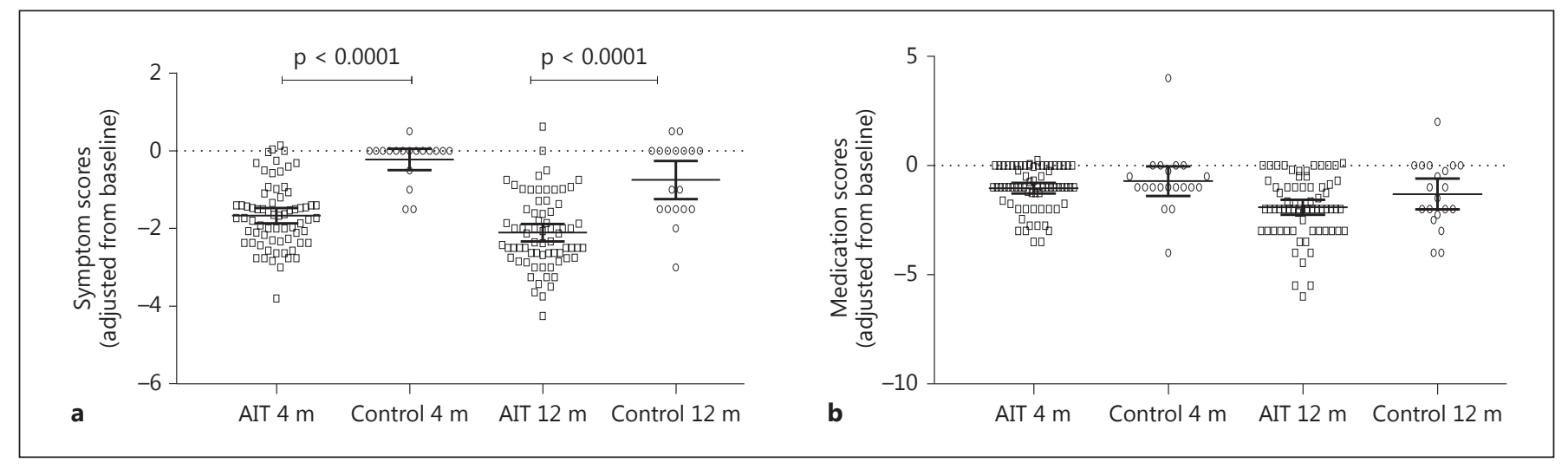

Fig. 1. The clinical symptom and medication scores of the AIT group compared with the control group during 1 year of AIT: symptom scores (a); medication scores (b). The data are presented by the change from baseline (after treatment - before treatment); the horizontal bars show the mean with $95 \%$ confidence limits. $4 \mathrm{~m}=4 \mathrm{months}$ of AIT; $12 \mathrm{~m}=12$ months of AIT.

nose blocking, running nose and sneezing) according to the severity, frequency of the symptoms and disturbance of daily activities and sleep in the previous week before their visit. The scores were evaluated in the range of $0-3(0=$ none, $1=$ mild, $2=$ moderate, $3=$ severe). The daily score was the sum of all symptoms in a day, and the mean score over 7 days was the score of the week.

Medication scores were calculated by assigning a score based on 1-500 $\mu \mathrm{g}$ of budesonide or the equivalent dose of inhaled corticosteroid, or $130 \mu \mathrm{g}$ of budesonide or the equivalent dose of nasal corticosteroid, each puff of salbutamol/terbutaline or the equivalent dose of another inhaled $\beta_{2}$-agnoist, and $10 \mathrm{mg}$ of oral loratidine or the equivalent dose of another antihistamine tablet. The daily score was the sum of all the medication used in a day, and the mean 7-day score was taken as the score for the week. The medication scores were only used to investigate the change during treatment, and were not used for the correlation with immunological parameters. The symptom and medication scores are reported as the change from baseline (after treatment - before treatment) in both the AIT and control groups.

\section{Immunological Tests}

Dp-specific IgE was measured using the ADVIA Centaur ${ }^{\circledR}$ immunoassay system (Siemens AG, Erlangen, Germany), which is a reverse sandwich immunoassay using direct chemiluminescent technology [18]. The IgE BF against Dp was measured on the Centaur ${ }^{\circledR}$ system using two different assay procedures: one is normal IgE determination which includes a wash step after serum incubation for removing all serum components except the bound $\operatorname{IgE}$ before adding the biotinylated antigen; the other is 'competition' IgE determination which excludes the wash step after serum incubation, and allows the non-IgE antibodies to compete with IgE in binding to biotinylated antigen. The $\operatorname{IgE} \mathrm{BF}=1-\left(\mathrm{IgE}_{\text {competition }} /\right.$ $\left.\operatorname{IgE}_{\text {normal }}\right)$. Dp-specific IgG4 in the serum samples was measured by a four-layer sandwich ELISA as reported previously [5].

\section{Statistical Analysis}

Samples with IgG4 results below the lower limit of quantification were reported as $20 \mathrm{AU} / \mathrm{ml}$, corresponding to half of the lim- it of quantification. A one-way ANOVA nonparametric test was used for comparing the difference between each pair of the three time points. Spearman's rank correlation test was used for studying the correlation between tested parameters. GraphPad Prism 6.0 for Windows (GraphPad Software, La Jolla, Calif., USA) was used for the statistical analysis in this study. A level of $\mathrm{p}<0.05$ was defined as significantly significant. The median value was used for each immunological response group as the data were not normally distributed. JMP ${ }^{\circledR} 11.0 .0$ (SAS institute Inc. U.S) was used for baseline adjustment analysis $[19,20]$ to test if the change of parameters were different between the active and control groups by taking the covariance into account.

\section{Results}

The demographic data, symptom and medication scores and antibody levels for the 69 patients in the AIT group and 20 patients in the open control group are shown in table 1 . There was no difference between the open control group and the AIT group in gender and medication scores, IgE, IgG4 and IgE BF. The mean values for age and symptom scores were slightly higher in the active group.

The use of pharmacotherapy was unrestricted in both groups and the children in the AIT group were treated with the recommended maintenance dose. All subjects completed the study.

\section{Changes in Symptom and Medication Scores}

Figure 1 shows the reduction of symptom and medication scores from baseline for the AIT and control groups after 4 and 12 months of AIT treatment. Before AIT, the 


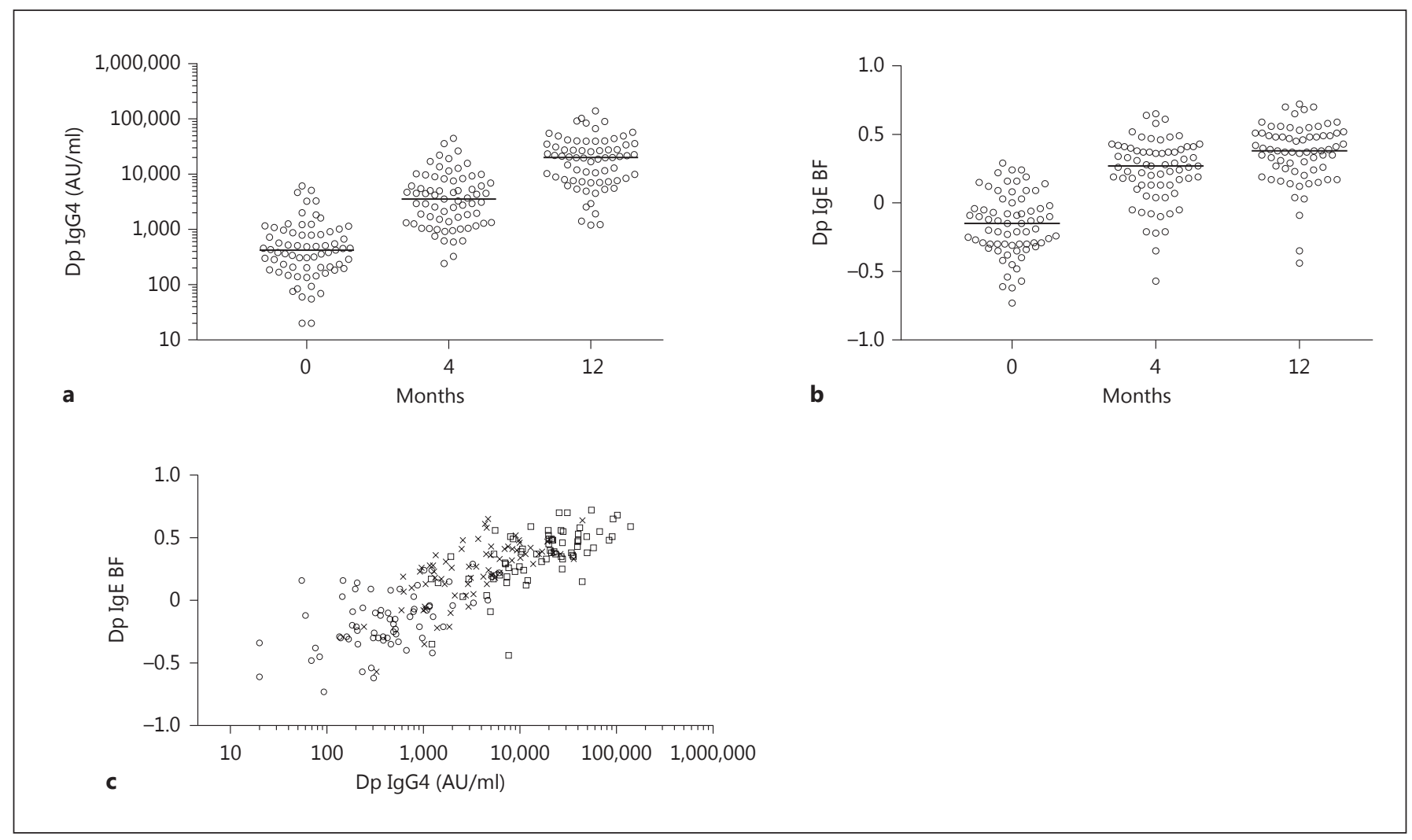

Fig. 2. The immunological responses and their correlation in the AIT group during 1 year of AIT. The horizontal bars show the median value. a Dp IgG4; the y-axis is a log scale. b Dp IgE BF. c The correlation between Dp IgE BF and Dp IgG4 at different time points. Circles represent pre-AIT (Spearman's $\mathrm{r}=0.433, \mathrm{p}=$ 0.0002 ); crosses indicate 4 months of AIT (Spearman's $r=0.680$, $\mathrm{p}<0.0001$ ); squares show 12 months of AIT (Spearman's $r=0.636$, $\mathrm{p}<0.001)$. mean value of the symptom scores for the AIT group was 3.0 compared to 2.0 for the control group (table 1) and $90 \%$ of AIT patients had symptom scores above 1.8 . The AIT group showed a statistically significant greater reduction in symptom scores after 4 and 12 months of AIT ( $p<0.0001$ for both time points) compared to the control group. The reduction in symptom scores in the AIT group was higher during the first 4 months of AIT compared to the period between 4 and 12 months of AIT ( $p<0.0001$, paired $t$ test). No such difference was observed in the control group.

There was no statistical difference observed in medication scores between the AIT and control groups after 4 and 12 months of AIT. However, 19 patients reported no use of symptomatic medication after 12 months of AIT compared to 5 patients before the start of AIT. For the control group, 5 patients reported no use of medication after 12 months compared to 2 patients before the start of treatment.

\section{Concentrations of Dp-Specific IgG4 and IgE}

All subjects in the AIT group had detectable levels of Dp-specific IgG4 before the start of AIT ranging from 20 to $6,108 \mathrm{AU} / \mathrm{ml}$ (fig. 2a). Concentrations of Dp-specific IgG4 showed a significant increase after 4 months of AIT $(\mathrm{p}<0.0001)$ and continued to increase during treatment. All AIT patients showed an increase in IgG4 concentrations after 4 months. The magnitude of increase in the specific IgG4 concentration was greater during the first 4 months of AIT compared to the increase between 4 and 12 months of AIT. There was no statistical difference in Dp-specific IgE before and after 12 months of AIT (data not shown).

\section{Changes in Dp IgE BF}

The ability of AIT-induced serum components to prevent binding between specific $\mathrm{IgE}$ and $\mathrm{Dp}$ allergens was measured in a competition assay and quantified as an allergen-specific IgE BF. No Dp IgE blocking activity could 

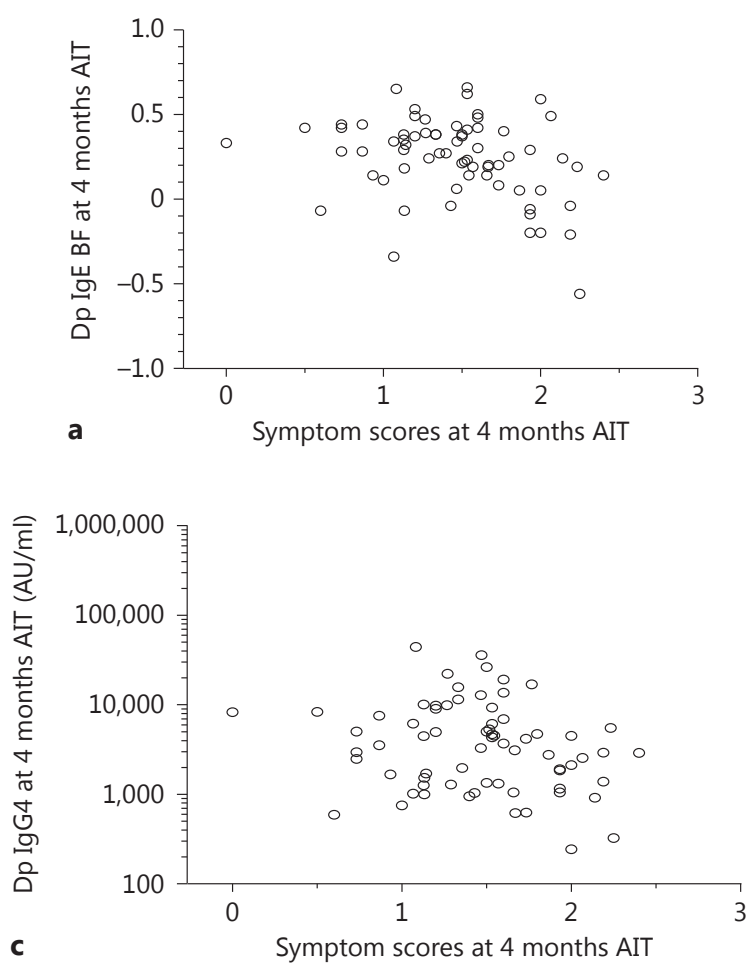

Fig. 3. The correlation between immunological and clinical response at 4 and 12 months of AIT. a Dp IgE BF versus symptom scores at 4 months of AIT (Spearman's $\mathrm{r}=-0.307, \mathrm{p}=0.010$ ). b Dp IgE BF versus symptom scores at 12 months of AIT (Spear-
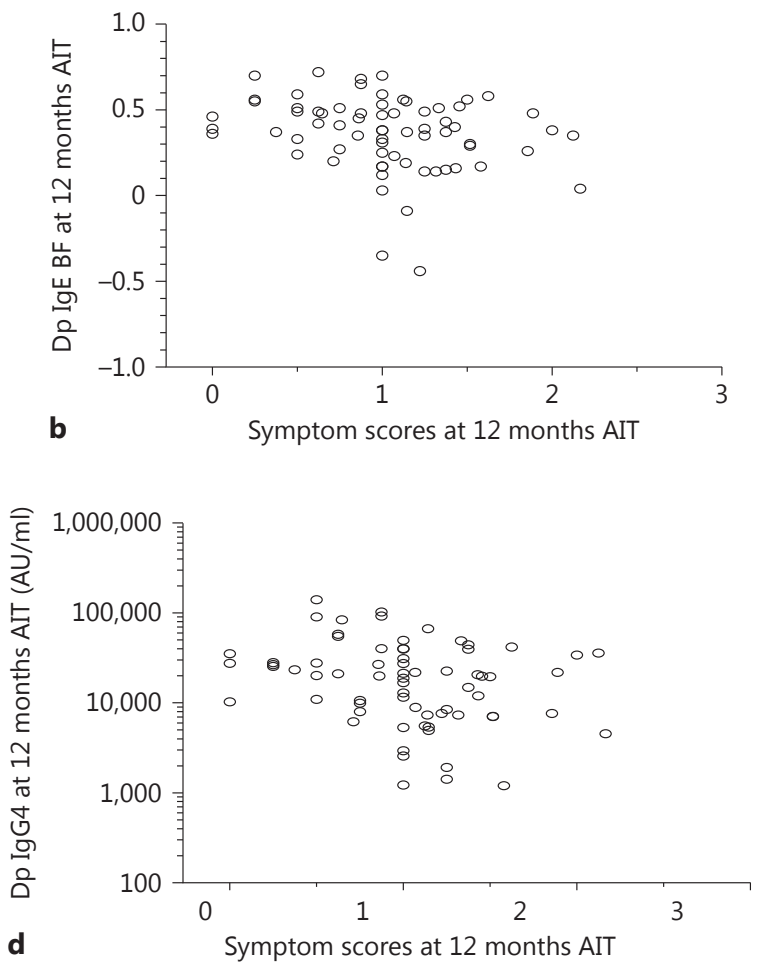

man's $r=-0.288, p=0.016)$. $c$ Dp IgG4 versus symptom scores at 4 months of AIT (Spearman's $r=-0.201, p=0.098)$. d Dp IgG4 versus symptom scores at 12 months of AIT (Spearman's $\mathrm{r}=$ $-0.296, \mathrm{p}=0.013)$. be detected in patients before AIT; however, there was a significant increase in blocking activity after 4 months of $\operatorname{AIT}(p<0.0001$; fig. 2b). The magnitude of increase of $\operatorname{IgE}$ blocking activity was larger during the first 4 months of AIT compared to the increase between 4 and 12 months of AIT. After 12 months of AIT, 78\% of subjects had an IgE BF higher than 0.2.

For the open control group, the ANOVA test showed no difference in symptom scores, Dp IgG4 concentrations or Dp IgE BF activity during the 12-month study period (data not shown).

\section{Correlation between Concentrations of IgG4, IgE BF and Clinical Symptoms}

The Dp IgE BF was positively correlated with Dp IgG4 concentrations at all time points, increasing progressively during the duration of AIT (before AIT: $\mathrm{r}=0.433, \mathrm{p}=$ $0.0002 ; 4$ months of AIT: $r=0.680, p<0.0001 ; 12$ months of AIT: $r=0.636, p<0.0001$; fig. $2 c)$. There was a weak but significant correlation between IgE $\mathrm{BF}$ and symptom scores both at 4 months of AIT $(r=-0.307, p=0.010$; fig. 3a) and after 12 months of AIT $(r=-0.288, p=0.016$; fig. 3b). There was a weak but significant correlation between Dp IgG4 levels and symptom scores after 12 months of $\operatorname{AIT}(r=-0.296, p=0.013$; fig. $3 c$ ) but not at 4 months of $\operatorname{AIT~}(r=-0.201, p=0.098$; fig. 3d).

\section{Clinical Improvement Predictability of Immunological} Responses at 4 Months of AIT

The ability of IgE BF and Dp IgG4 levels at 4 months of AIT to predict a clinical improvement at 12 months of AIT was investigated using the correlation with symptom scores at 4 and 12 months of AIT. There was a significant difference in symptom score at 12 months of AIT between the patients having an $\operatorname{IgE} B F \geq 0.2$ at 4 months of AIT compared to patients with an IgE BF $<0.2(p=0.0093$; fig. 4). No significant correlation was found between symptom scores and Dp IgG4 levels. 


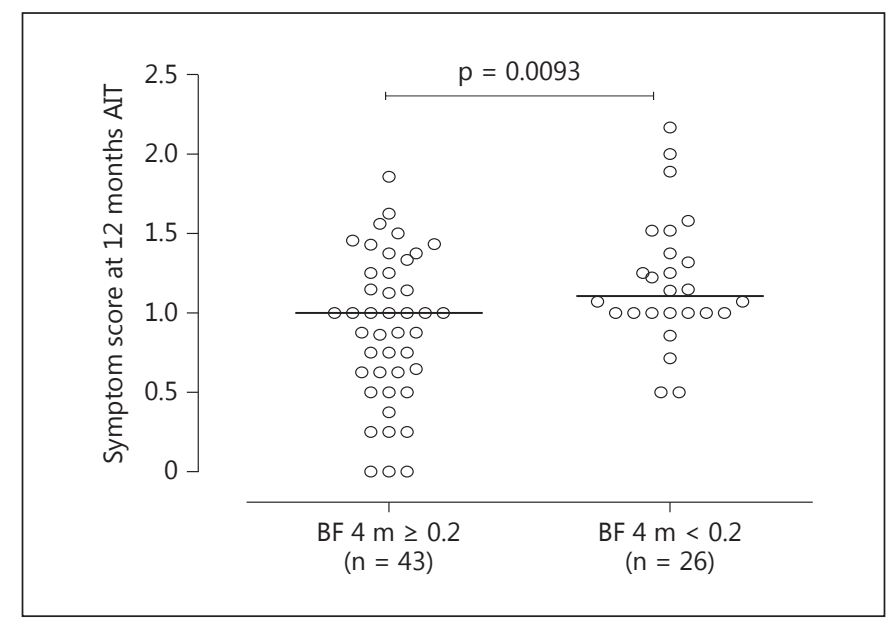

Fig. 4. The symptom scores at 12 months grouped by the level of $\mathrm{Dp}$ IgE BF at 4 months of AIT (BF $4 \mathrm{~m}$, cutoff $=0.2)$. The horizontal bars show the median level in each group.

\section{Discussion}

In this study we investigated the correlations between clinical symptoms and specific antibody responses in dust mite-allergic children undergoing AIT under reallife conditions. Due to ethical and practical considerations, the study could not be conducted as a randomized, double-blind, placebo-controlled trial. However, using the same treatment product and protocol, the clinical efficacy of subcutaneous AIT in Chinese mite-allergic children has already been demonstrated in a randomized, double-blind, placebo-controlled study [21]. The aim of this study was to address the existence of previously identified biomarkers among children undergoing HDM AIT. To our knowledge, this is the first time AIT-induced biomarkers have been demonstrated in mite-allergic children. Although the real-life conditions somewhat restrict the study design, we still believe the findings are important for generating hypotheses regarding clinical and immunological AIT correlations that can be tested under more stringent clinical study conditions. Allergic symptoms before and during AIT were recorded and there were no attempts to control or taper the use of symptomatic medication. During our study conditions, children in the AIT group had higher symptom scores compared to the control group before the initiation of AIT and showed a progressive reduction in symptoms during the course of AIT starting from the end of the up-dosing phase compared to the open control group. This finding is in agree- ment with Wang et al. [21], who reported a significant reduction in symptom scores in the AIT group compared to the placebo group. We also observed an increase of $20 \%$ of AIT patients that reported no need to use symptomatic medication to control their allergic symptoms after 12 months of AIT. However, the use of pharmacotherapy was unrestricted and we did not observe any significant difference in medication use between the AIT and control groups which could be due to the study duration being limited to 12 months. The median age in the control group was lower than in the AIT group. However, matching the groups by removing selected children in the control group did not change the significance of our finding. In agreement with many other studies [5, 14, 22-24], we found that AIT induced a substantial increase of allergen-specific IgG4 antibodies and we also found a weak but significant correlation between concentrations of IgG4 and symptoms after 12 months of AIT. Thus, in addition to serving as an early marker for a specific immune response towards the administrated vaccine, levels of AIT-induced IgG4 could reflect a clinical improvement during later stages of AIT.

It has been shown for pollen AIT that functional IgGassociated inhibitory activity, as determined by the IgE BF assay and inhibition of FcRII (CD23)-dependent binding of allergen-IgE complexes to B cells, correlates with a clinical improvement $[14,25]$. In this study we measured the ability of AIT-induced serum components to inhibit the binding between specific IgE and HDM allergens quantified as the allergen-specific IgE BF. We found a very strong and progressively increasing correlation between levels of HDM-specific IgG4 and the IgE BF during AIT, demonstrating that HDM allergen IgG4 contributes to the detected blocking activity. There was a weak but significant correlation between $\operatorname{IgE} B F$ and symptoms already after 4 months of AIT which was maintained at 12 months of AIT, supporting the suggestion that functional assays of inhibitory IgG4 and IgE BF may be more useful surrogates of clinical response than IgG4 [14].

Before AIT, the majority of patients have detectable allergen-specific IgG4, probably originating from natural exposure; however, there was no measurable blocking activity. The lack of IgE blocking activity despite the presence of IgG4 could be due to low IgG4 concentrations or low IgG4 affinity compared to specific IgE. The differences between the degree of increase of IgG4 and of IgE $\mathrm{BF}$ during the AIT up-dosing and maintenance phases indicates that, in addition to IgG4 concentrations, other factors such as clonality and affinity maturation also in- 
fluence the blocking activity [26]. In addition to high levels of specific IgG4, HDM immunotherapy might result in the production of functionally active high-affinity allergen-specific blocking IgG4, as demonstrated for pollen AIT by James et al. [27].

The recommended duration of AIT is at least 3 years; therefore, we investigated to what degree the IgE BF at an early stage of AIT could predict later symptom improvements at a later stage. We found that the patients with an IgE BF above 0.2 after 4 months AIT showed significantly fewer symptoms at 12 months compared to patients with $\mathrm{BF}$ values below 0.2 . We could not find any similar predictive correlations for levels of IgG4. Although the correlation was weak, it indicates that IgG4-associated functional measurements at an early stage of AIT could have a predictable value for AIT clinical outcomes. Whether or not functional IgG-associated assays can be used as surrogates reflecting and predicting AIT clinical outcomes requires further investigation in larger randomized, placebo-controlled studies.

\section{Conclusions}

During AIT, a significant decrease in allergic symptoms was observed in HDM-allergic children. The levels of AIT-induced specific IgG4 correlate with the inhibition of specific IgE binding to HDM allergen as measured by the allergen IgE BF assay. The IgE BF significantly correlates with symptoms after 4 and 12 months of AIT. A high IgE BF value at an early stage of AIT is associated with lower symptom scores at a later stage of AIT.

\section{Acknowledgements}

The authors thank Tiantian Liu and Haifeng Zhong at ALKGIRD lab, Guangzhou, China, for excellent technical assistance. We also thank Henrik Ipsen at ALK in Denmark for the statistical consultation, and Prof. Stephan Durham for critically reviewing the manuscript.

\section{References}

1 Larenas-Linnemann DES, Pietropaolo-Cienfuegos DR, Calderón MA: Evidence of effect of subcutaneous immunotherapy in children: complete and updated review from 2006 onward. Ann Allergy Asthma Immunol 2011; 107:407-416.

-2 Erekosima N, Suarez-Cuervo C, Ramanathan M, Kim JM, Chelladurai Y, Segal JB, Lin SY: Effectiveness of subcutaneous immunotherapy for allergic rhinoconjunctivitis and asthma: a systematic review. Laryngoscope 2014; 124:616-627.

- 3 Jacobsen L, Niggemann B, Dreborg S, Ferdousi HA, Halken S, Høst A, Koivikko A, Norberg LA, Valovirta E, Wahn U, Möller C; THE PAT investigator group: Specific immunotherapy has long-term preventive effect of seasonal and perennial asthma: 10-year follow-up on the PAT study. Allergy 2007;62: 943-948.

4 Aalberse RC, van der Gaag R, van Leeuwen J: Serological aspects of IgG4 antibodies. I. prolonged immunization results in a IgG4-restricted response. J Immunol 1983;130:722726.

5 Lai X, Li J, Xiao X, Liu E, Zhang C, Wang H, Gjesing B, Zhong N, Spangfort MD: Specific IgG4 production during house dust mite immunotherapy among age, gender and allergic disease populations. Int Arch Allergy Immunol 2013;160:37-46.

6 Wachholz PA, Durham SR: Mechanisms of immunotherapy: IgG revisited. Curr Opin Allergy Clin Immunol 2004;4:313-318.
7 van Neerven RJ, Wikborg T, Lund G, Jacobsen B, Brinch-Nielsen A, Arnved J, Ipsen H: Blocking antibodies induced by specific allergy vaccination prevent the activation of CD4+ T cells by inhibiting serum-IgE-facilitated allergen presentation. J Immunol 1999; 163:2944-2952.

8 Wachholz PA, Kristensen Soni N, Till SJ, Durham SR: Inhibition of allergen-IgE binding to B cells by IgG antibodies after grass pollen immunotherapy. J Allergy Clin Immunol 2003;112:915-922.

-9 van Neerven RJ, Arvidsson M, Ipsen H, Sparholt SH, Rak S, Würtzen PA: A double-blind, placebo controlled birch allergy vaccination study: inhibition of CD23-mediated serum immunoglobulin E-facilitated allergen presentation. Clin Exp Allergy 2004;34:420-428.

10 Würtzen PA, Lund G, Lund K, Arvidsson M, Rak S, Ipsen H: A double-blind placebo-controlled birch allergy vaccination study II: correlation between inhibition of IgE binding, histamine release and facilitated allergen presentation. Clin Exp Allergy 2008;38:1290-1301.

11 van der Zee JS, van Swieten P, Aalberse RC: Inhibition of complement activation by IgG4 antibodies. Clin Exp Immunol 1986;64:415422.

12 Aalberse RC, Stapel SO, Schuurman J, Rispers T: Immunoglobulin G4: an odd antibody. Clin Exp Allergy 2009;39:469-477.

$\checkmark 13$ Flicker S, Valenta R: Renaissance of the blocking antibody concept in type I allergy. Int Arch Allergy Immunol 2003;132:13-24.
14 Shamji MH, Ljørring C, Francis JN, Calderon MA, Larché M, Kimber I, Frew AJ, Ipsen H, Lund K, Würtzen PA, Durham SR: Functional rather than immunoreactive levels of $\mathrm{IgG}_{4}$ correlate closely with clinical response to grass pollen immunotherapy. Allergy 2012; 67:217-226.

15 James LK, Shamji MH, Walker SM, Wilson DR, Wachholz PA, Francis JN, Jacobson MR, Kimber I, Till SJ, Durham SR: Long-term tolerance after allergen immunotherapy is accompanied by selective persistence of blocking antibodies. J Allergy Clin Immunol 2011; 127:509-516.e5.

16 Li J, Sun B, Huang Y, Lin X, Zhao D, Tan G, Wu J, Zhao H, Cao L, Zhong N; China Alliance of Research on Respiratory Allergic Disease: A multicenter study assessing the prevalence of sensitizations in patients with asthma and/or rhinitis in China. Allergy 2009;64: 1083-1092.

17 Francis JN, James LK, Paraskevopoulos G, Wong C, Calderon MA, Durham SR, Till SJ: Grass pollen immunotherapy: IL-10 induction and suppression of late responses precedes IgG4 inhibitory antibody activity. J Allergy Clin Immunol 2008;121:1120-1125.e2.

18 Petersen AB, Gudmann P, Gronager PM, Morkeberg R, Bogestrand S, Linneberg A and Johansen N: Performance evaluation of a specific IgE assay developed for the ADVIA Centaur $^{\mathbb{B}}$ immunoassay system. Clin Biochem 2004;37:882-892. 
19 Glymour MM, Weuve J, Berkman LF, Kawachi I, Robins JM: When is baseline adjustment useful in analyses of change? An example with education and cognitive change. Am J Epidemiol 2005;162:267-278.

20 Garofolo KM, Yeatts SD, Ramakrishnan V, Jauch EC, Johnston KC, Durkalski VL: The effect of covariate adjustment for baseline severity in acute stroke clinical trials with responder analysis outcomes. Trials 2013;14:98.

21 Wang H, Lin X, Hao C, Zhang C, Sun B, Zheng J, Chen P, Sheng J, Wu A, Zhong N: A double-blind, placebo-controlled study of house dust mite immunotherapy in Chinese asthmatic patients. Allergy 2006;61:191-197.

22 Blumberga G, Groes L, Dahl R: SQ-standardized house dust mite immunotherapy as an immunomodulatory treatment in patients with asthma. Allergy 2011;66:178-185.
23 Pevec B, Radulovic Pevec M, Stipic Markovic A, Batista I, Rijavec M, Silar M, Kosnik M, Korosec P: House dust mite-specific immunotherapy alters the basal expression of $\mathrm{T}$ regulatory and FceRI pathway genes. Int Arch Allergy Immunol 2012;159:287-296.

24 Yukselen A, Kendirli SG, Yilmaz M, Altintas DU, Karakoc GB: Effect of one-year subcutaneous and sublingual immunotherapy on clinical and laboratory parameters in children with rhinitis and asthma: a randomized, placebo-controlled, double-blind, double-dummy study. Int Arch Allergy Immunol 2012; $157: 288-298$
25 Shamji MH, Wilcock LK, Wachholz PA, Dearman RJ, Kimber I, Wurtzen PA, Larché M, Durham SR, Francis JN: The IgE-facilitated allergen binding (FAB) assay: validation of a novel flow-cytometric based method for the detection of inhibitory antibody responses. J Immunol Methods 2006;317:71-79.

26 Holm J, Willumsen N, Wurtzen PA, Christensen LH, Lund K: Facilitated antigen presentation and its inhibition by blocking IgG antibodies depends on IgE repertoire complexity. J Allergy Clin Immunol 2011;127: 1029-1037.

27 James LK, Bowen H, Calvert RA, Dodev TS, Shamji MH, Beavil AJ, McDonnell JM, Durham SR, Gould HJ: Allergen specificity of IgG4-expressing B cells in patients with grass pollen allergy undergoing immunotherapy. J Allergy Clin Immunol 2012;130:663-670.

\section{Erratum}

In the article by Zhao et al., entitled 'The functional IgE-blocking factor induced by allergen-specific immunotherapy correlates with IgG4 antibodies and a decrease of symptoms in house dust mite-allergic children' [Int Arch Allergy Immunol 2016;169:113-120, DOI: $10.1159 / 000444391]$, the first affiliation should read:

Respiratory Department, The Affiliated Nanjing Children's Hospital of Nanjing Medical

University, Nanjing 